\title{
Assessment of Mental Health Problems of School Children Aged 11-17 Years Using Self Report Strength and Difficulty Questionnaire (SDQ)
}

\author{
Rimal HS ${ }^{1}$, Pokharel $A^{2}$ \\ ${ }^{1}$ Dr. Hem Sagar Rimal, MBBS, MD, Fellowship training \\ in Developmental Pediatrics (Australia), Department of \\ Paediatrics, ${ }^{2}$ Archana Pokharel, Lecturer, Department \\ of Psychology. Both from Nobel Medical College and \\ Teaching Hospital, Biratnagar, Nepal.
}

\section{Introduction}

It is now recognized that psychological disorders, among children and adolescents have high prevalence rate. Over the past decades the public health relevance of mental health conditions in children and adolescents has been of growing concern for everyone ${ }^{1}$. In the last century, there had been considerable changes in the nature and pattern of health problems affecting children, and one of the most striking of these changes is the growing importance of mental disorders as a source of childhood morbidity ${ }^{2}$. Currently, there is limited data on the prevalence of mental health problems among adolescents especially in the developing country like Nepal. The Strengths and Difficulties Questionnaire (SDQ) is one of the most commonly used instruments for screening psychopathology in children and adolescents ${ }^{3}$. There is a consensus opinion that children and adolescents living with adversities and the experience of psychosocial difficulties are more vulnerable to have psychological and behavioral problems. But these inferences are mostly taken from the researches done in affluent and developed countries ${ }^{4,5,6}$.

\section{Materials and Methods}

Participants were School children of grade six, seven, eight and nine, aged 11-17 years from one of the private school in Biratnagar where the medium of instruction was English. Pretested questionnaire were administered to the target students. Data was also collected about the educational and occupational status of both the parents.

\author{
Address for correspondence \\ Dr. Hem Sagar Rimal \\ Department of Paediatrics \\ Nobel Medical College and Teaching Hospital \\ Biratnagar, Nepal \\ E-mail: hemsagarr@yahoo.com
}

\begin{abstract}
Introduction: It is now recognized that psychological disorders, among children and adolescents have high prevalence rate. Currently, there is limited data on the prevalence of mental health problems among adolescents especially in the developing country like Nepal. The objective of this study was to investigate the prevalence of mental health issues among school children aged 11-17 years. Materials and Methods: The self rated version of Strength and Difficulty Questionnaire (SDQ-YR1) were given to the students and answers were collected and the data was analyzed using SPSS version 16.1. The questions were in the Likert scale 0 (not true), 1 (somewhat true) and 2(certainly true). The data collected also included parent's educational and occupational status. Results: A total of 159 students participated in the study after receiving well informed consent from the parents. There were $96(60 \%)$ boys and $63(40 \%)$ girls' participants in the study. Among all the participants in this study $18.6 \%$ had a total-Strength and Difficulty Questionnaire (SDQ) score that can be classified as abnormal by published cutoffs. The emotional problems $(24.5 \%)$ and peer relationship problem $(22 \%)$ were the two common problems screened as an abnormal SDQ score. Girls were significantly more likely to have emotional problems than boys ( $p$ value $<0.05$ ) where as boys were significantly more likely to have Hyperactivity/inattention score in SDQ than girls ( $p$ value $<0.05$ ). Gender difference was also significant statistically as girls had higher abnormal total SDQ score than boys ( $p$ value < $0.05)$. Conclusion: There is high prevalence of mental health issues in children but a study in large sample is recommended.
\end{abstract}

Key words: School children, SDQ, Mental health, emotional problem, ADHD, Conduct disorder.
How to cite this article?

Rimal HS, Pokharel A. Assessment of Mental Health Problems of School Children Aged 11-17 Years Using Self Report Strength and Difficulty Questionnaire (SDQ). J Nepal Paediatr Soc 2013;33(3):172-176. 
The following tools were used: The Strength and Difficulties Questionnaire (SDQ).

The SDQ is an instrument that has been widely used to assess mental health problems, emotional and behavioral problems and strength among children and adolescents $^{7}$. The final conclusion on the presence or absence of mental health problems as measured by $S D Q$ is ideally computed from the combined reports from parents, teachers, and self-report by child ${ }^{8}$. However, self reports may be sufficient screening tool for adolescents aged 11 years or older ${ }^{9}$. The clinical usefulness of SDQ in identifying mental health problems in adolescents has been established, with a reliability and validity that is as good as that of Child Behavior Checklist ${ }^{10}$. The Self rated SDQ possesses 25 items in the following 5 -item scales: emotional and conduct problems, hyperactivity/ inattention, peer relationship problems, and pro-social behavior. Each item is scored on a 3-point scale ( 0 not true; 1 somewhat true; 2 certainly true) and the sum of all answered items in a scale creates its total score (possible range, $0-10$ ), whereas the sum of all answered items in the first 4 scales creates the total overall score (possible range, 0-40). The higher the total score is the larger the difficulties are. The SDQ total scores could be considered as "normal" (range, 0-15), "borderline" (range, 16-19), and "abnormal" (range, 17-40), indicating the presence of general psychopathology. For the subscales, abnormal scores were taken as follows: emotional scale and hyperactivity/inattention range, 7 to 10; conduct problems range, 5 to 10 ; peer relationship problems range, 6 to 10; and pro-social behavior range, 0 to 4 . The abnormal SDQ score in any area indicate substantial risk of clinically significant problem in that area $^{11}$.

The diagnostic predilection: The information provided by respondents is used to predict how likely a young person is to have emotional, behavioral or concentration problems severe enough to warrant a diagnosis according to the ICD-10 or DSM-IV classifications. For each diagnostic grouping, there are three possible predictions: 'low risk', 'medium risk' and 'high risk'. In general, these predictions agree fairly well with what an expert would say after a detailed assessment of the young person. Around $25-60 \%$ of young people who are rated as 'high risk' do turn out to have the relevant diagnosis according to experts. So do around $10-15 \%$ of 'medium risk' young people but only about $1-4 \%$ of 'low risk' young people.

All adolescents were informed about the aims and procedures of the study. Those participants who were 16 years and older signed consent forms whereas the younger participants got their consent form signed by their parents and returned to the data collector.

\section{Results}

\section{General Sample Characteristics}

A total of 159 children and adolescent participated in the study and successfully completed the self rated Strength and Difficulty Questionnaire with impact supplement (YRS). The mean age of the participant was 14.11 years with minimum age of 11 years and maximum of 17 years. There is preponderance of male students $96(60 \%)$ and female participants were 63 (40\%). Regarding employment status $93 \%$ of fathers were employed where as $28 \%$ of mothers were unemployed.

Table 1: Characteristics of the sample population:

\begin{tabular}{|l|c|}
\hline \multicolumn{1}{|c|}{ Variables } & $\mathbf{N}=159(100 \%)$ \\
\hline Gender & $96(60.4 \%)$ \\
Fale & $63(39.6 \%)$ \\
\hline Grades of students & \\
Grade six & $13(8 \%)$ \\
Grade seven & $48(30 \%)$ \\
Grade eight & $14(9 \%)$ \\
Grade nine & $30(19 \%)$ \\
Grade ten & $54(34 \%)$ \\
\hline Employment status of mother & \\
Employed & $44(28 \%)$ \\
Unemployed & $115(72 \%)$ \\
\hline Employment status of Father & \\
Employed & $148(93 \%)$ \\
Unemployed & $11(7 \%)$ \\
\hline
\end{tabular}

\section{Self rated mental health status}

A mean of $26.4 \%$ of all the adolescents, in this study, had a total-Strength and Difficulty Questionnaire (SDQ) score that can be classified as abnormal by published cutoffs. There was statistically significant gender difference noted across the domain of impact of the difficulties in young person's life, where girls were significantly more likely to have impact of difficulties ( $p$ value < 0.05). The percentage of students having an abnormal SDQ score across emotional problem domain was 24.5 while it was $22 \%$, noted across peer relationship problem area.

Girls were significantly more likely to have emotional problems than boys ( $p$ value $<0.05$ ). In contrast to this, boys were significantly more likely to have Hyperactivity/inattention score in SDQ than girls ( $p$ value $<0.05$ ). Gender difference was also significant statistically in terms of girls having high abnormal total SDQ score. The gender difference was not statistically significant in the domain of conduct problem, peer relationship problem and pro-social behavior. 
The data was analyzed using official online software to find out the predicting capacity of the SDQ to meet mental health diagnosis meeting DSM-IV criteria. The prediction of having any mental health diagnosis in the study population was $20.1 \%$, which means there is a $25-60 \%$ chance of them receiving any of the DSM-IV mental health diagnosis if evaluated by an expert.

\section{Discussion}

This study investigated the prevalence of mental health illnesses in the school children and adolescents aged 11-17 years using the Strength and Difficulty questionnaire. Our study population comprised of $60 \%$ boys and $40 \%$ girls. The percentage of fathers who were employed was ninety three but only twenty eight percentages of mothers were employed.

As it was a pilot study in a relatively small sample size, it has its limitations to generalize its finding to the whole population; still the findings of this study are worth discussing as there is no such study done in Nepal to look at mental health status of school children. This study has clearly indicated that there is high prevalence (25\%) of mean value of abnormal total-Strength and Difficulty Questionnaire (SDQ) score as per cut off set by different studies. Olyanka et al evaluated the prevalence, pattern, and socio-demographic correlates of self-reported mental health problems among a pilot sample of adolescents from 5 developing countries. In that study the prevalence of mean abnormal total difficulty score was $10.5 \%(5.8-15)$ and this value was lower than the findings of our study where it is $25 \%{ }^{12}$.

There are few studies around the world where higher prevalence rates of abnormal total SDQ scores have been reported and our study has findings comparable to those studies. In a study done in Brazil, a total abnormal SDQ score prevalence was $18 \%$ and In Iran it was $20 \%$. $^{13 \cdot 14}$ There was another study done in Andhra Pradesh, India where $22.43 \%$ of children had abnormal SDQ at least in one domain ${ }^{15}$.

We noted high abnormal SDQ scores across the two domains of emotional problems $(24.5 \%)$ and peer relationship problem (22\%). There is statistically significant gender difference noted in case of abnormal rating in the area of emotional problem and total abnormal SDQ score, as girls rating higher than the boys ( $p$ value $<0.05$ ). Dutch girls had significantly higher values on the emotional symptoms and pro-social behavior subscale, while boys scored higher on the conduct problems scale ${ }^{16}$. Similarly, in a study done in Finland where girls of grade $7^{\text {th }}$ and $9^{\text {th }}$ scored much higher than boys on emotional symptoms and pro social behavior ${ }^{17}$.

Table 2: Gender wise Distribution of Clinical Ranges of (SDQ) Scores among Participants

\begin{tabular}{|c|c|c|c|}
\hline \multirow{2}{*}{ SDQ Clinical Ranges (Dichotomized) } & \multicolumn{3}{|c|}{ Gender Distribution ( $n=159$ ) } \\
\hline & Male & Female & $p$ value \\
\hline $\begin{array}{l}\text { Emotional problem score } \\
\text { Normal/borderline (n 120; 75.5\%) } \\
\text { Abnormal (n 39; } 24.5 \% \text { ) }\end{array}$ & $\begin{array}{l}78(81 \%) \\
18(19 \%)\end{array}$ & $\begin{array}{l}38(51 \%) \\
18(49 \%)\end{array}$ & $<0.05^{*}$ \\
\hline $\begin{array}{l}\text { Hyperactivity/inattention score } \\
\text { Normal/borderline (n 131; } 82.4 \% \text { ) } \\
\text { Abnormal (n } 28 ; 11.6 \% \text { ) }\end{array}$ & $\begin{array}{l}74(84 \%) \\
22(16 \%)\end{array}$ & $\begin{array}{c}57(90.5 \%) \\
6(9.5 \%) \\
\end{array}$ & $\begin{array}{c}<0.05 \\
*\end{array}$ \\
\hline $\begin{array}{l}\text { Conduct problem score } \\
\text { Normal/borderline (n 133; 83.6\%) } \\
\text { Abnormal (n } 26 ; 16.4 \%)\end{array}$ & $\begin{array}{l}81(84 \%) \\
15(16 \%) \\
\end{array}$ & $\begin{array}{c}57(90.5 \%) \\
6(9.5 \%) \\
\end{array}$ & 0.27 \\
\hline $\begin{array}{l}\text { Peer relationship problem score } \\
\text { Normal/borderline (n 124; 78\%) } \\
\text { Abnormal (n 35; } 22 \%)\end{array}$ & $\begin{array}{l}78(81 \%) \\
18(19 \%) \\
\end{array}$ & $\begin{array}{l}46(73 \%) \\
17(27 \%) \\
\end{array}$ & 0.22 \\
\hline $\begin{array}{l}\text { Pro-social score } \\
\text { Normal/borderline (n 147; 92.4\%) } \\
\text { Abnormal (n } 12 ; 7.6 \%)\end{array}$ & $\begin{array}{l}86(89.5 \%) \\
10(10.5 \%)\end{array}$ & $\begin{array}{c}61(97 \%) \\
2(3 \%)\end{array}$ & 0.09 \\
\hline $\begin{array}{l}\text { Difficulty with young person's life } \\
\text { Normal/borderline (n 121; 76\%) } \\
\text { Abnormal (n 38; } 24 \%)\end{array}$ & $\begin{array}{l}78(81 \%) \\
18(19 \%)\end{array}$ & $\begin{array}{l}43(68 \%) \\
20(32 \%)\end{array}$ & 0.06 \\
\hline $\begin{array}{l}\text { Total score } \\
\text { Normal/borderline (n 119; 69\%) } \\
\text { Abnormal (n 40; 25\%) }\end{array}$ & $\begin{array}{l}79(87.5 \%) \\
17(12.5 \%)\end{array}$ & $\begin{array}{l}40(56 \%) \\
23(44 \%)\end{array}$ & $\begin{array}{c}<0.05 \\
*\end{array}$ \\
\hline
\end{tabular}

${ }^{*} p$ value $<0.05$ indicates significant at $\mathrm{Cl}$ of $95 \%$. 
In our study the boys have rated higher than girls in the domain of hyperactivity and inattention which is statistically significant ( $p$ value $<0.05$ ). These findings are similar to the findings from a study done in Pakistani school children where boys had higher estimates of behavior or externalizing problems but emotional problems were found more amongst girls ${ }^{18}$. This finding of gender differences in the prevalence of mental health problem in children and adolescent is different from the findings in other studies ${ }^{19}$. Ravens-Sieberer et al reported that gender differences in the prevalence rates of mental health problems among children depend on children ages, with differences more apparent in the younger age groups ${ }^{20}$.

This study highlights that there is a need to screen school children and adolescent for mental health problems, not identifying and addressing these problems may have detrimental effect on their academic performance and on potential adult life. In developing country like Nepal mental health disorders can be a product of disadvantaged societies, poverty, political unrest, violence of all kinds and adverse living condition of the vulnerable children and adolescent. ${ }^{14}$

\section{Conclusion}

This pilot study has demonstrated that there is high prevalence of mental health problems in school going children and adolescents. As there was small sample size in this study, question can be raised about its external validity. Hence further research with adequate sample size using appropriate tools is recommended.

Limitations: A major limitation to this study is that the reports from parents and teachers were not taken and the sample population was not very representative as well. There is a need for some systemic studies that will explore the prevalence of mental health problems in Nepal, which will help to guide our future mental health planning.

Acknowledgement: The authors want to acknowledge the support from Dharanidharan Baral, statistician from BPKIHS for the analysis of data. Authors are also grateful to all the school children who participated in this study and the teachers of the school.

Funding: None

Conflict of interest: None

Permission from IRB: Yes

\section{References}

1. Ravens-Sieberer U, Wille N, Erhart M, Bettge S, Wittchen HU, Rothenberger A, et al. Prevalence of mental health problems among children and adolescents in Germany: results of the BELLA study within the National Health Interview and Examination Survey. Eur Child Adoles Psychiatry 2008;17 Suppl 1:22-33.

2. Palfrey JS TT, Green M, et al. Introduction: addressing the millennial morbidity-the context of community. Pediatrics 2005; 115:1121-3.

3. He JP, Burstein M, Schmitz A, Merikangas KR. The Strengths and Difficulties Questionnaire (SDQ): the factor structure and scale validation in U.S. adolescents. J Abnorm Child Psychol 2013; 41(4):583-95.

4. Barbarin OA RL. Adversity and psychosocial competence of South African children. Am J Orthopsychiatry 1999; 69.319-27.

5. ML. R. Psychosocial adversity and child psychopathology. Brit J Psychiatry Res 1999;174:480-93.

6. Hackett $\mathrm{R} \mathrm{HL}$, Bhakta $\mathrm{P}$, et.al.Life events in a South Indian population and their association with psychiatric disorder in children. Int J Soc Psychiatry 2000(46):201-7.

7. G. R. The Strengths and Difficulties Questionnaire: a research note. J Child Psychol Psychiatry 1997(38):581-6.

8. Goodman R MH, Bailey V. The Strengths and Difficulties Questionnaire: a pilot study on the validity of the self-report version. Eur Child Adolesc Psychiatry 1998; 7:125-30.

9. Muris $P$ MC, van Den Berg F. The Strengths and Difficulties Questionnaire (SDQ): further evidence for its reliability and validity in a community sample of Dutch children and adolescents. Eur Child Adolesc Psychiatry 2003;12:1-8.

10. Goodman R SS. Comparing the Strengths and Difficulties Questionnaire and the Child Behavior Checklist: is small beautiful? 1999. J Abnorm Child Psychol 1999; 27:17-24.

11. R. G. Psychometric properties of the Strengths and Difficulties Questionnaire. J Am Acad Child Adolesc Psychiatry 2001;40:1337-345.

12. Atilola O, Singh Balhara YP, Stevanovic D, Avicenna M, Kandemir H. Self-reported mental health problems among adolescents in developing countries: results from an international pilot sample. J Dev Behav Pediatr 2013;34(2):129-37.

13. Cury CR GJ. Strengths and difficulties questionnaire (SDQ): a study of school children in Ribeirão Preto. Rev Bras Psiquiatr 2003;25:139-45.

14. Arman S KM, Maracy MR, et al. Epidemiological study of youth mental health using Strengths and Difficulties Questionnaire (SDQ). Iran Red Crescent Med J 2012; 14:371-5. 
15. Bele SD, Bodhare TN, Valsangkar S, Saraf A. An epidemiological study of emotional and behavioral disorders among children in an urban slum. Psychol Health Med 2013; 18(2):223-32.

16. Muris $P$ MC, van Den Berg F. The Strengths and Difficulties Questionnaire (SDQ): further evidence for its reliability and validity in a community sample of Dutch children and adolescents. Eur Child Adolesec Psychiatry (2003);12:1-8.

17. Koskelainen M SA, Vauras M. Self-reported strengths and difficulties in a community sample of Finnish adolescents. Eur Child Adolesc Psychiatry Res 2001;10:180-5.
18. Syed EU, Hussein SA, Haidry SE. Prevalence of emotional and behavioural problems among primary school children in Karachi, Pakistan--multi informant survey. Indian J Pediatr 2009;76(6):6237.

19. Cortina MA SA, Fazel M, et.al. Prevalence of child mental health problems in sub-Saharan Africa: a systematic review. Arch Pediatr Adolesc Med 2012(166):276-81.

20. Ravens-Sieberer U, Erhart M, Gosch A, Wille $\mathrm{N}$. Mental health of children and adolescents in 12 European countries-results from the European KIDSCREEN study. Clinical psychology \& psychotherapy. 2008;15(3):154-63. Epub 2008/12/31 